\title{
IVAN PAN
}

\section{MARCOS SEBASTIANI}

\section{Classification des feuilletages turbulents}

Annales de la faculté des sciences de Toulouse $\sigma^{e}$ série, tome 12, no 3 (2003), p. 395-413

<http://www.numdam.org/item?id=AFST_2003_6_12_3_395_0>

(C) Université Paul Sabatier, 2003, tous droits réservés.

L'accès aux archives de la revue «Annales de la faculté des sciences de Toulouse » (http://picard.ups-tlse.fr/ annales/) implique l'accord avec les conditions générales d'utilisation (http://www.numdam.org/conditions). Toute utilisation commerciale ou impression systématique est constitutive d'une infraction pénale. Toute copie ou impression de ce fichier doit contenir la présente mention de copyright.

\section{NumDam}

Article numérisé dans le cadre du programme Numérisation de documents anciens mathématiques http://www.numdam.org/ 


\title{
Classification des feuilletages turbulents ${ }^{(*)}$
}

\author{
IVAN PAN ${ }^{(1)}$, MARCos SEBASTIANI ${ }^{(2)}$
}

\begin{abstract}
RÉSUMÉ. - Soit $h: X \rightarrow B$ une fibration elliptique relativement minimale. On démontre que l'ensemble des feuilletages turbulents sur $X$ (dans le sens de Brunella) dont le diviseur de tangence avec la fibration est donné, possède une structure naturelle de variété analytique connexe ; on calcule la dimension de cette variété.
\end{abstract}

\begin{abstract}
Let $h: X \rightarrow B$ be a relatively minimal elliptic fibration. We proof that the set of turbulent foliations on $X$ (on Brunella's sense) which has a fixed tangence divisor with the fibration is, in a natural way, a connected analytic variety; we also calculate its dimension.
\end{abstract}

\section{Introduction}

Soit $h: X \rightarrow B$ une fibration elliptique relativement minimale de la surface analytique compacte connexe $X$. Soit $\Lambda$ un feuilletage turbulent de $X$ avec fibration adaptée $h$ (cette notion a été introduite par M. Brunella dans $[3$, chap. $4, \S 3]$; voir aussi $[2, \S 5])$. Notons $D$ le diviseur de tangence de $\Lambda$ avec le feuilletage $\Gamma$ défini par la fibration $h$ (voir $[2, \S 1]$ ).

On considère la famille $\mathcal{F}_{\Lambda}$ des feuilletages analytiques à singularités isolées de $X$ dont le diviseur de tangence avec $\Gamma$ est encore $D$ (ces feuilletages sont aussi turbulents avec fibration adaptée $h$ ).

Soient $T$ le fibré tangent à $\Lambda([5, \S 2])$ et $T_{X}$ le fibré tangent à $X$. Alors $\mathcal{F}_{\Lambda}$ s'identifie de façon naturelle avec un sous-ensemble de l'espace projectif $\mathbb{P}\left(\operatorname{Hom}\left(T, T_{X}\right)\right)$ associé à l'espace vectoriel des homomorphismes de $T$

(*) Reçu le 2 décembre 2002, accepté le 8 janvier 2003

(1) Instituto de Matemática, UFRGS, av. Bento Gonçalves 9500, 91540-000 Porto Alegre, RS, Brasil.

E-mail: pan@mat.ufrgs.br

(2) Instituto de Matemática, UFRGS, av. Bento Gonçalves 9500, 91540-000 Porto Alegre, RS, Brasil.

E-mail: sebast@mat.ufrgs.br 
dans $T_{X}([5, \S 2]$ et lemme 3.5 plus bas). Dans ce qui suit on démontre, en introduisant un invariant qui classifie les éléments de $\mathcal{F}_{\Lambda}$, que l'adhérence de $\mathcal{F}_{\Lambda}$ dans $\mathbb{P}\left(\operatorname{Hom}\left(T, T_{X}\right)\right)$ est un sous-espace linéaire dont on calcule explicitement la dimension en termes de $D$ (théorème 5.4 , corollaire 5.5 et propositions 6.5 et 6.6 ).

Cela généralise [4] en suivant les orientations d'Étienne Ghys à qui nous exprimons ici notre reconnaissance.

\section{Notations}

Soit $X$ une variété analytique complexe et soit $\mathcal{O}_{X}$ son faisceau structurel. Si $E$ est un fibré vectoriel holomorphe sur $X$, on note $\mathcal{O}_{X}(E)$ le $\mathcal{O}_{X}$-module de ses sections holomorphes. Si $D$ est un diviseur de $X$, on note $\mathcal{O}_{X}(D)$ le $\mathcal{O}_{X}$-module dont les sections sur un ouvert $U \subset X$ sont les fonctions méromorphes $f$ sur $U$ telles que $\operatorname{div} f+\left.D\right|_{U} \geqslant 0$. On peut associcr à $D$ un fibré vectoriel holomorphe $E_{D}$ de rang 1 tel que $\mathcal{O}_{X}\left(E_{D}\right) \cong \mathcal{O}_{X}(D)$. Si $\mathcal{A}$ est un $\mathcal{O}_{X}$-module et $D$ un diviseur de $X$ on note

$$
\mathcal{A}(D):=\mathcal{A} \otimes \mathcal{O}_{X} \mathcal{O}_{X}(D) .
$$

La caractéristique d'Euler-Poincaré arithmétique de $X$ est

$$
\chi(X):=\sum_{j}(-1)^{j} \operatorname{dim}_{\mathbb{C}} H^{j}\left(X, \mathcal{O}_{X}\right),(X \text { compacte }) .
$$

On désigne par $K_{X}$ le fibré canonique de $X$. Si $E$ est un fibré vectoriel sur $X$, on dénote $E_{x}$ la fibre sur $x \in X$. Si $Z$ est un espace analytique irréductible, $\mathbb{C}[Z]$ et $\mathbb{C}(Z)$ désignent l'anneau des fonctions holomorphes et le corps des fonctions méromorphes sur $Z$ respectivement.

Finalement, si $\mathbb{C}^{*}:=\mathbb{C}-\{0\}$ et $V$ est un $\mathbb{C}$-espace vectoriel on note $\mathbb{P}(V):=(V-\{0\}) / \mathbb{C}^{*}$ l'espace projectif associé à $V$.

Le foncteur « image réciproque » sera consideré dans le sens de [1, chap. I, §8].

\section{Feuilletages analytiques des surfaces}

Soit $X$ une surface analytique complexe connexe. Un feuilletage analytique $\Lambda$ de $X$ est défini par un couple $(T, \varphi)$ où $T$ est un fibré vectoriel holomorphe de dimension 1 sur $X$ et $\varphi: T \rightarrow T_{X}$ est un homomorphisme non-nul. Un autre couple $\left(T^{\prime}, \varphi^{\prime}\right)$ définit aussi $\Lambda$ si et seulement s'il existe un isomorphisme $\theta: T \rightarrow T^{\prime}$ tel que $\varphi=\varphi^{\prime} \circ \theta$. C'est-à-dire, dans le cas 
où $T=T^{\prime}$, si et seulement s'il existe $g: X \rightarrow \mathbb{C}^{*}$ holomorphe telle que $\varphi\left|T_{x}=g(x) \varphi^{\prime}\right| T_{x}$ pour tout $x \in X$.

L'ensemble singulier de $\Lambda$ est l'ensemble

$$
\text { Sing } \Lambda:=\left\{x \in X: \varphi \mid T_{x}=0\right\} .
$$

C'est un sous-ensemble analytique de dimension $\leqslant 1$. Si $\operatorname{dim} \operatorname{Sing} \Lambda=0$ ou Sing $\Lambda=\emptyset$, on dit que $\Lambda$ est à singularités isolées (voir $[5, \S 1]$ ).

LEMME 3.1. - Supposons que $X$ est compacte et soient $\Lambda=(T, \varphi), \Lambda^{\prime}=$ $\left(T, \varphi^{\prime}\right)$ deux feuilletages de $X$. Supposons de plus que:

a) $\Lambda$ est à singularités isolées ;

b) il existe un ouvert non-vide $U \subset X$ tel que $\varphi\left(T_{x}\right)=\varphi^{\prime}\left(T_{x}\right)$ pour tout $x \in U$.

Alors $\Lambda=\Lambda^{\prime}$.

Preuve. - Considérons l'ouvert

$$
\left.V:=X-\left[(\operatorname{Sing} \Lambda) \cup \operatorname{Sing} \Lambda^{\prime}\right)\right] .
$$

On peut supposer $U \subset V$. Les images des homomorphismes $\varphi, \varphi^{\prime}$ définissent deux sections holomorphes du fibré en droites projectives associé à $T_{V}$. Comme ces sections coïncident au-dessus de $U$, elles sont identiques. Donc, il existe $g: V \rightarrow \mathbb{C}^{*}$ holomorphe telle que

$$
\varphi^{\prime}\left|T_{x}=g(x) \varphi\right| T_{x}, x \in V .
$$

Soit $a \in X-V$. Si $U_{a}$ est un voisinage ouvert de $a$ tel qu'il existe une section holomorphe et jamais nulle $s$ de $T$ sur $U_{a}$, alors

$$
\varphi^{\prime}(s(x))=g(x) \varphi(s(x)), x \in U_{a} \cap V .
$$

L'hypothèse a) implique que la restriction de $g$ à $U_{a} \cap V$ se prolonge à une fonction holomorphe sur $U_{a}$. On en déduit que $g$ se prolonge à une fonction holomorphe sur $X$ tout entier. Donc $g$ est constante.

DÉfinition 3.2. - Soit $\Lambda=(T, \varphi)$ un feuilletage analytique de $X$. Notons

$$
\varphi_{*}: \mathcal{O}_{X}(T) \rightarrow \mathcal{O}_{X}\left(T_{X}\right)=: \xi_{X}, \varphi^{*}: \Omega_{X}:=\mathcal{O}_{X}\left(T_{X}^{\vee}\right) \rightarrow \mathcal{O}_{X}\left(T^{\vee}\right)
$$

les homomorphismes des faisceau induits par $\varphi$. On appelle

$$
\mathcal{T}_{\Lambda}:=\operatorname{Im} \varphi_{*} \subset \xi_{X} \text { et } \mathcal{N}_{\Lambda}^{\vee}:=\operatorname{Ker} \varphi^{*} \subset \Omega_{X}
$$


les faisceaux tangent et conormal à $\Lambda$ respectivement. (Ce sont des $\mathcal{O}_{X}$ modules localement libres de rang 1. Comme $\varphi_{*}$ est injectif, on peut supposer $\mathcal{T}_{\Lambda}=\mathcal{O}_{X}(T)$ ). Les faisceaux cotangent et normal à $\Lambda$ sont $\mathcal{T}_{\Lambda}^{\vee}$ et $\mathcal{N}_{\Lambda}:=$ $\left(\mathcal{N}_{\Lambda}^{\vee}\right)^{\vee}$ respectivement.

LEMme 3.3. - Si $\Lambda$ est à singularités isolées, alors

$$
\mathcal{T}_{\Lambda}^{\vee} \otimes \mathcal{O}_{X} \mathcal{N}_{\Lambda}^{\vee} \cong \mathcal{O}_{X}\left(K_{X}\right)
$$

en tant que $\mathcal{O}_{X}$-modules.

Preuve. - Voir [3, chap. 2, §1].

Dans ce qui suit, si $\Lambda, \Gamma$ sont deux feuilletages de $X$, on dira qu'ils sont différents $(\Lambda \neq \Gamma)$ si leur restrictions à $X-(\operatorname{Sing} \Lambda \cup \operatorname{Sing} \Gamma)$ ne sont pas identiques (comparer avec 3.1).

Soient $\Lambda \neq \Gamma$ deux feuilletages de $X$. On a une application évidente

$$
\mathcal{T}_{\Lambda} \otimes \mathcal{O}_{X} \mathcal{N}_{\Gamma}^{\vee} \rightarrow \mathcal{O}_{X}
$$

C'est un homomorphisme injectif de $\mathcal{O}_{X}$-modules. Donc, son image est de la forme $\mathcal{O}_{X}(-D)$ où $D$ est un diviseur effectif.

DÉfinition 3.4. $-D=: \operatorname{tang}(\Lambda, \Gamma)$ est le diviseur de tangence de $\Lambda$ et $\Gamma([2, \S 1])$.

LEMME 3.5. - On a les assertions suivantes :

a) Si $\Lambda$ et $\Gamma$ sont à singularités isolées, alors $\operatorname{tang}(\Lambda, \Gamma)=\operatorname{tang}(\Gamma, \Lambda)$.

b) $\mathcal{O}_{X}(\operatorname{tang}(\Lambda, \Gamma))=\mathcal{T}_{\Lambda}^{\vee} \otimes_{\mathcal{O}_{X}} \mathcal{N}_{\Gamma}$.

c) Si $\Lambda_{1}=\left(T_{1}, \varphi_{1}\right)$ et $\Lambda_{2}=\left(T_{2}, \varphi_{2}\right)$ sont des feuilletages différents de $\Gamma$ tels que $\operatorname{tang}\left(\Lambda_{1}, \Gamma\right)=\operatorname{tang}\left(\Lambda_{2}, \Gamma\right)$, alors $T_{1} \cong T_{2}$.

Preuve. - L'assertion c) suit de b) tandis que celle-ci suit de la définition 3.4. Pour a) voir $[2, \S 1]$.

\section{Fibrations elliptiques}

Soit $h: X \rightarrow B$ une fibration elliptique relativement minimale de la surface analytique compacte connexe $X$. Pour chaque $y \in B$, on désigne par $h^{-1}(y)$ l'ensemble analytique réduit, support de la fibre $X_{y}$ de $h$ sur $y$; on pose

$$
\begin{gathered}
X_{y}=\sum_{j} k_{y j} C_{y j}, \\
-398-
\end{gathered}
$$


où les $C_{y j}$ dénotent les composantes irréductibles de $h^{-1}(y)$. Notons $m_{y}:=$ $p g d c_{j}\left\{k_{y j}\right\}$ la multiplicité de $X_{y}$; posons $k_{y j}^{\prime}:=k_{y j} / m_{y}$.

DÉFINITION 4.1. - On définit le diviseur effectif

$$
M:=\sum_{y} \sum_{j}\left(k_{y j}^{\prime}-1\right) C_{y j}
$$

de $X$ (voir [3, chap.2, §3]).

Par la suite $\Gamma$ désignera le feuilletage défini par la fibration $h$.

LEMME 4.2. - On a un isomorphismes de $\mathcal{O}_{X}$-modules

$$
\mathcal{T}_{\Gamma} \cong h^{*}\left[h_{* 1}\left(\mathcal{O}_{X}\right)\right] \otimes \mathcal{O}_{X} \mathcal{O}_{X}(M)
$$

Preuve. - Voir [3, chap. 2, §3].

LEMME 4.3. - $h_{* 1}\left(\mathcal{O}_{X}\right)$ est un $\mathcal{O}_{B}$-module localement libre de rang 1.

Preuve. - Il suit de [1, cor. III, (11.2)] compte tenu du fait que la fibre générique est elliptique.

LEMME 4.4. - L'homomorphisme canonique $\mathcal{O}_{B} \rightarrow h_{*}\left(\mathcal{O}_{X}(M)\right)$ obtenu par composition avec $h$ est un isomorphisme de $\mathcal{O}_{B}$-modules.

Preuve. - Soit $U \subset B$ un ouvert ; posons $V:=h^{-1}(U)$. Comme $h$ est propre et à fibres connexes, toute $f \in \mathbb{C}(V)$ dont les pôles sont des composantes des fibres est de la forme $f=g$ oh pour une $g \in \mathbb{C}(U)$. Si $g$ a un pôle en $y \in U$, alors $f$ a un pôle d'ordre $\geqslant k_{y j}$ en $C_{y j}$. Puisque $k_{y j}>k_{y j}^{\prime}-1$, on voit que $f \in H^{0}\left(V, \mathcal{O}_{X}(M)\right)$ si et seulement si $g \in H^{0}\left(U, \mathcal{O}_{B}\right)$.

LeMme 4.5. - Soit $y \in B$. Alors il existe un ouvert $U \subset B$ tel que $y \in U$ et $\mathcal{T}_{\Gamma}\left|V \cong \mathcal{O}_{X}(M)\right| V$ en tant que $\mathcal{O}_{V}$-modules, où $V:=h^{-1}(U)$.

Preuve. - Soit $U \ni y$ un ouvert de $B$ tel que $h_{* 1}\left(\mathcal{O}_{X}\right) \mid U \cong \mathcal{O}_{U}$ (lemme). Alors

$$
h^{*}\left(h_{* 1}\left(\mathcal{O}_{X}\right)\right)\left|V \cong \mathcal{O}_{V}=\mathcal{O}_{X}\right| V,
$$

et on applique le lemme 4.2 .

- Lemme 4.6. - On a un isomorphisme de $\mathcal{O}_{B}$-modules

$$
h_{*}\left(\mathcal{T}_{\Gamma}\right) \cong h_{* 1}\left(\mathcal{O}_{X}\right)
$$


Preuve. - Soit $U \subset B$ un ouvert ; posons $V:=h^{-1}(U)$. L'application

$$
H^{0}\left(U, h_{* 1}\left(\mathcal{O}_{X}\right)\right) \rightarrow H^{0}\left(V, h^{*}\left(h_{* 1}\left(\mathcal{O}_{X}\right) \otimes_{\mathcal{O}_{X}} \mathcal{O}_{X}(M)\right)\right.
$$

définie par $s \mapsto h^{*}(s) \otimes 1$ induit un homomorphisme de $\mathcal{O}_{B}$-modules

$$
h_{* 1}\left(\mathcal{O}_{X}\right) \longrightarrow h_{*}\left(h^{*}\left(h_{* 1}\left(\mathcal{O}_{X}\right)\right) \otimes_{\mathcal{O}_{X}} \mathcal{O}_{X}(M)\right)
$$

qui est un isomorphisme d'après les lemmes 4.3 et 4.4 .

L'assertion suit du lemme 4.2.

Lemme 4.7. - Pour tout $y \in B$ il existe un voisinage ouvert $U \subset B$ de $y$ et un champ de vecteurs holomorphe $v$ sur $V:=h^{-1}(U)$ tangent aux fibres de h tel que:

a) $v$ s'annule sur $C_{y j}$ avec multiplicité $k_{y j}^{\prime}-1$;

b) si $w$ est un champ de vecteurs holomorphe sur $V$ tangent aux fibres de $h$, alors $w=(g \circ h) v$ pour une $g \in \mathbb{C}[U]$;

c) $v$ ne s'annule pas sur $V-h^{-1}(y)$.

Preuve. - On choisit $U$ comme dans le lemme 4.5 ; on a $\mathcal{T}_{\Gamma}\left|V \cong \mathcal{O}_{X}(M)\right|$ $V$. D'après le lemme 4.4, $H^{0}\left(V, \mathcal{O}_{V}(M)\right)$ est engendré par 1 comme $H^{0}$ $\left(U, \mathcal{O}_{B}\right)=\mathbb{C}[U]$-module. En tant que section de $\mathcal{O}_{X}(M)$ sur $V$, l'élément 1 s'annule sur $C_{y j}$ avec multiplicité $k_{y j}^{\prime}-1$. Donc $H^{0}\left(V, \mathcal{T}_{\Gamma}\right)$ est engendré, en tant que $\mathbb{C}[U]$-module, par une section qui s'annule sur $C_{y j}$ avec cette multiplicité.

\section{Feuilletages turbulents}

Soit $h: X \rightarrow B$ une fibration elliptique relativement minimale de la surface analytique compacte connexe $X$; désignons par $\Gamma$ le feuilletage associé à $h$. Soit $\Lambda=(T, \varphi)$ un feuilletage turbulent de $X$ avec fibration adaptée $h$ (dans le sens de [3, chap. $4, \S 3]$; en particulier $\Lambda$ est à singularités isolées). Alors le diviseur de tangence $D:=\operatorname{tang}(\Lambda, \Gamma)$ est de la forme

$$
D=\sum_{y, j} t_{y j} C_{y j}
$$

où les $t_{y j}$ sont des entiers $\geqslant 0$ (on garde les notations du $\S 4$ ).

DÉFINITION 5.1. - On définit le diviseur effectif

$$
\begin{aligned}
R: & =\sum_{y \in B} r_{y} \cdot y \\
& -400-
\end{aligned}
$$


de $B$ par

$$
r_{y}:=\inf _{j}\left[\frac{t_{y j}+k_{y j}+k_{y j}^{\prime}-2}{k_{y j}}\right],
$$

où le crochet dénote la « partie entière » d'un nombre rationnel.

Observons que $R$ ne dépend que de $D$.

Considérons maintenant l'homomorphisme de $\mathcal{O}_{X}$-modules

$\mu: h^{*}\left(\Omega_{B}(R) \otimes_{\mathcal{O}_{B}} h_{*}\left(\mathcal{T}_{\Gamma}\right)\right) \longrightarrow \mathcal{N}_{\Gamma}^{\vee} \otimes_{\mathcal{O}_{X}} \mathcal{T}_{\Gamma} \otimes_{\mathcal{O}_{X}} \mathcal{O}_{X}(D)=\left(\mathcal{N}_{\Gamma}^{\vee} \otimes_{\mathcal{O}_{X}} \mathcal{T}_{\Gamma}\right)(D)$

défini par

$$
h^{*}(\omega \otimes v) \mapsto h^{*}(\omega) \otimes v \otimes 1,
$$

où $\omega \in H^{0}\left(U, \Omega_{B}(R)\right)$ et $v \in H^{0}\left(V, \mathcal{T}_{\Gamma}\right)$ avec $U$ un ouvert de $B$ et $V=$ $h^{-1}(U)$. Observons que $\omega$ a, au plus, un pôle d'ordre $\leqslant r_{y}$ en $y \in U$. Alors $h^{*}(\omega)$ a un pôle d'ordre $\leqslant k_{y j} r_{y}-\left(k_{y j}-1\right)$ le long de $C_{y j}$. Donc, d'après le lemme $4.7, h^{*}(\omega) \otimes v$ a un pôle le long de $C_{y j}$ d'ordre, au plus

$$
\begin{aligned}
k_{y j} r_{y}-\left(k_{y j}-1\right)-\left(k_{y j}^{\prime}-1\right) & =k_{y j} r_{y}-\left(k_{y j}+k_{y j}^{\prime}-2\right) \\
& \leqslant t_{y j} .
\end{aligned}
$$

Du fait que tous les faisceaux en question sont localement libres on déduit tout de suite que $\mu$ est injectif.

D'autre part, considérons l'homomorphisme de $\mathcal{O}_{X}$-modules

$$
\lambda:\left(\mathcal{N}_{\Gamma}^{\vee} \otimes_{\mathcal{O}_{X}} \mathcal{T}_{\Gamma}\right)(D) \longrightarrow \mathcal{H o m}\left(T, T_{X}\right)
$$

défini par

$$
\lambda(\eta \otimes v)(s)=\eta(\varphi(s)) \cdot v,
$$

où $\eta$ est une 1-forme méromorphe sur un ouvert $V \subset X$ nulle sur les fibres de $h$ et telle que $f \eta$ est holomorphe si $f \in H^{0}\left(V, \mathcal{O}_{X}(-D)\right)$, $v$ est un champ de vecteurs holomorphe sur $V$ tangent aux fibres de $h$ et $s$ est une section holomorphe de $T$ au-dessus de $V$. Observons que $\eta(\varphi(s))$ est holomorphe par définition de $D$.

Comme $\Lambda \neq \Gamma$, le lemme 3.1 nous dit que $\lambda$ est injectif.

Finalement, $\lambda \circ \mu$ induit une application $\mathbb{C}$-linéaire injective au niveau des sections globales

$$
(\lambda \circ \mu)_{*}: H^{0}\left(B, \Omega_{B}(R) \otimes_{\mathcal{O}_{B}} h_{*}\left(\mathcal{T}_{\Gamma}\right)\right) \longrightarrow \operatorname{Hom}\left(T, T_{X}\right) .
$$


DÉfinition 5.2. - Posons $E(D):=H^{0}\left(B, \Omega_{B}(R) \otimes h_{*}\left(\mathcal{T}_{\Gamma}\right)\right)$. Pour chaque $\alpha \in E(D)$ on définit le feuilletage de $X$ suivant :

$$
\Lambda_{\alpha}:=\left(T, \varphi_{\alpha}\right), \text { où } \varphi_{\alpha}:=\varphi+(\lambda \circ \mu)_{*}(\alpha) .
$$

Remarque 5.3. - Comme $\Lambda$ est génériquement transverse aux fibres de $h$, on voit tout de suite que $\varphi_{\alpha} \neq 0$ et que $\Lambda_{\alpha} \neq \Gamma$. En effet, par définition, pour tout $\alpha \in E(D)$ l'image de l'homomorphisme $(\lambda \circ \mu)_{*}(\alpha): T \rightarrow T_{X}$ est engendré par des vecteurs tangents aux fibres de $h$.

THÉORÈME 5.4. - On a les assertions suivantes :

a) Pour tout $\alpha \in E(D)$ on a $\operatorname{tang}\left(\Lambda_{\alpha}, \Gamma\right)=D$; en particulier si $\Lambda_{\alpha}$ est à singularités isolées, alors $\Lambda_{\alpha}$ est turbulent avec fibration adaptée $h$.

b) Si $\alpha, \beta \in E(D)$ avec $\alpha \neq \beta$, alors $\Lambda_{\alpha} \neq \Lambda_{\beta}$.

c) Si $\Lambda^{\prime}$ est un feuilletage turbulent de $X$ avec fibration adaptée $h$ tel que tang $\left(\Lambda^{\prime}, \Gamma\right)=D$, alors il existe $\alpha \in E(D)$ tel que $\Lambda_{\alpha}=\Lambda^{\prime}$.

d) L'ensemble des $\alpha \in E(D)$ tels que $\Lambda_{\alpha}$ est à singularités isolées est le complémentaire de la réunion d'un nombre fini de sous-variétés linéaires propres.

Preuve. - a) Soit $U \subset X$ un ouvert et soient $s$ et $\omega$ des sections holomorphes de $T$ et $\mathcal{N}_{\Gamma}^{\vee}$ au-dessus de $U$ respectivement. Comme $(\lambda \circ \mu)_{*}(\alpha)(s)$ est un champ de vecteurs sur $U$ tangent aux fibres de $h$, on a $\omega(\varphi(s))=\omega\left(\varphi_{\alpha}(s)\right)$. Alors

$$
\operatorname{tang}\left(\Lambda_{\alpha}, \Gamma\right)=\operatorname{tang}(\Lambda, \Gamma)=D
$$

b) Si $\Lambda_{\alpha}=\Lambda_{\beta}$, alors il existe $c \in \mathbb{C}^{*}$ tel que

$$
\varphi+(\lambda \circ \mu)_{*}(\alpha)=c\left(\varphi+(\lambda \circ \mu)_{*}(\beta)\right),
$$

d'où suit

$$
(1-c) \varphi=(\lambda \circ \mu)_{*}(c \beta-\alpha) .
$$

Or, prenons $y \in B$ en sorte que $X_{y}$ soit une fibre régulière transverse à $\Lambda$. Si $x \in h^{-1}(y)$, alors pour tout $u \in T_{x}$ le vecteur $(1-c) \varphi(u)$ est au même temps transverse et tangent à $h^{-1}(y)$, d'après la dernière égalité. Donc $c=1$. Puisque $(\lambda \circ \mu)_{*}$ est injectif, on en déduit $\alpha=\beta$.

c) On va définir $\alpha$ localement au voisinage, disons $U$, d'un point $y \in$ $B$. On peut supposer qu'il existe sur $V:=h^{-1}(U)$ un champ de vecteurs holomorphe $v$ qui s'annule sur $C_{y j}$ avec multiplicité $k_{y j}^{\prime}-1$ et qui est tangent aux fibres de $h$ (lemme 4.7a). 
Soit $u$ un champ de vecteurs holomorphe et jamais nul sur $U$. Si $U$ est suffisamment petit, $u \mid(U-\{y\})$ se relève à deux champs de vecteurs holomorphes $w, w^{\prime}$ sur $V-h^{-1}(y)$ tangents à $\Lambda, \Lambda^{\prime}$ respectivement. Il en résulte

$$
w^{\prime}-w=f \cdot v \mid\left(V-h^{-1}(y)\right), f \in \mathbb{C}(V) .
$$

Comme $h$ est propre et $f$ holomorphe sur $V-h^{-1}(y)$ on peut écrire $f=$ $g \circ h$ pour une fonction méromorphe $g \in \mathbb{C}(U)$. On définit une 1-forme méromorphe $\omega$ sur $U$ par

$$
\omega(u)=g
$$

C'est-à-dire :

$$
w^{\prime}=w+h^{*}(\omega)(w) \cdot v
$$

$\operatorname{sur} V-h^{-1}(y)$.

Affirmation. - Supposons que $g$ a un pôle d'ordre $r$ en $y$. Alors $r \leqslant r_{y}$.

On définit

$$
\alpha \mid U:=\omega \otimes v
$$

Il découle du lemme 4.7 et de l'affirmation ci-dessus que $\alpha$ est bien définie comme section de $\Omega_{B}(R) \otimes_{\mathcal{O}_{B}} h_{*}\left(\mathcal{T}_{\Gamma}\right)$.

On va prouver que $\Lambda_{\alpha}=\Lambda^{\prime}$. Par le lemme $3.5 \mathrm{c}$ on peut supposer $\Lambda^{\prime}=$ $\left(T, \varphi^{\prime}\right)$. D'après le lemme 3.1 , il suffit de prouver que $\varphi_{\alpha}\left(T_{x}\right)=\varphi^{\prime}\left(T_{x}\right)$ pour tout $x \in V-h^{-1}(y)$ dans le cas où $X_{y}$ est une fibre réguliére transverse à $\Lambda$ et $\Lambda^{\prime}$. Dans ce cas $w$ et $w^{\prime}$ sont holomorphes sur $V$ et $\omega$ est holomorphe sur $U$. Le champ $w$ définit une section holomorphe jamais nulle $s$ de $T \mid V$ telle que $\varphi(s)=w$. Alors,

$$
\begin{aligned}
\varphi_{\alpha}(s) & =\varphi(s)+(\lambda \circ \mu)_{*}(\alpha)(s) \\
& =w+\lambda_{*}\left(\mu_{*}(\alpha)\right)(s) \\
& =w+h^{*}(\omega)(\varphi(s)) \cdot v \\
& =w+h^{*}(\omega)(w) \cdot v \\
& =w^{\prime} .
\end{aligned}
$$

On en déduit l'assertion.

Preuve de l'affirmation. - Soient $\xi, \eta$ des coordonnées locales de $X$ centrées en un point générique de $C_{y j}$; pour simplifier écrivons $C=C_{y j}$ et $k=k_{y j}, k^{\prime}=k_{y j}^{\prime}, t=t_{y j}$ et $r^{\prime}=r_{y}$ : on va montrer $r \leqslant r^{\prime}$. Soit $z$ une coordonnée locale de $B$ centrée en $y$. On peut supposer que $h$ s'exprime en coordonnées locales par $z=\xi^{k}$. 
Par ailleurs on prend $u=\partial / \partial z$. On a

$$
\frac{\partial}{\partial \xi}=\frac{d z}{d \xi} \frac{\partial}{\partial z}=k \xi^{k-1} \frac{\partial}{\partial z}
$$

Donc on peut écrire

$$
w=k^{-1} \xi^{1-k} \frac{\partial}{\partial \xi}+c(\xi, \eta) \frac{\partial}{\partial \eta}
$$

et

$$
w^{\prime}=k^{-1} \xi^{1-k} \frac{\partial}{\partial \xi}+c^{\prime}(\xi, \eta) \frac{\partial}{\partial \eta}
$$

où $c, c^{\prime}$ sont holomorphes si $\xi \neq 0$ avec, au plus, un pôle en $\xi=0$.

D'autre part,

$$
v=\epsilon \xi^{k^{\prime}-1} \frac{\partial}{\partial \eta}
$$

où $\epsilon$ est holomorphe et non-nulle. Nous devons prouver

$$
k r \leqslant t+k+k^{\prime}-2 \text {. }
$$

Supposons d'abord que $t=0$. Comme par hypothèse $\Lambda$ et $\Lambda^{\prime}$ ont le même diviseur de tangence avec $\Gamma$, ces deux feuilletages sont transverses à $C$ au voisinage du point considéré. Cela implique que $c$ et $c^{\prime}$ ont, au plus, un pôle d'ordre $k-1$ en $\xi=0$. Puisque

$$
\begin{aligned}
w^{\prime}-w & =\left(c^{\prime}(\xi, \eta)-c(\xi, \eta)\right) \frac{\partial}{\partial \eta} \\
& =\left[\left(c^{\prime}(\xi, \eta)-c(\xi, \eta)\right) \xi^{1-k^{\prime}} \epsilon^{-1}\right] \cdot v
\end{aligned}
$$

on a que $f$ a, au plus, un pôle d'ordre $k+k^{\prime}-2$ le long de $C$; mais l'ordre de ce pôle est $k r$.

Supposons maintenant $t \neq 0$. Donc, ni $\Lambda$ ni $\Lambda^{\prime}$ ne sont transverses à $C$ au voisinage du point considéré ; d'où qu'il existe des entiers $p, q \geq k-1$ tels que

$$
w=k^{-1} \xi^{1-k} \frac{\partial}{\partial \xi}+\xi^{-p} b(\xi, \eta) \frac{\partial}{\partial \eta}
$$

et

$$
w^{\prime}=k^{-1} \xi^{1-k} \frac{\partial}{\partial \xi}+\xi^{-q} b^{\prime}(\xi, \eta) \frac{\partial}{\partial \eta}
$$


pour des fonctions holomorphes $b, b^{\prime}$ qui ne s'annulent pas sur $\xi=0$. Alors $\xi^{p} w$ et $\xi^{q} w^{\prime}$ sont des champs de vecteurs tangents à $\Lambda$ et $\Lambda^{\prime}$ respectivement, qui ne s'annulent pas sur $C$. Comme $\Gamma$ est localement défini par $d \xi=0$ et

$$
d \xi\left(\xi^{p} w\right)=k^{-1} \xi^{1-k+p}, d \xi\left(\xi^{q} w^{\prime}\right)=k^{-1} \xi^{1-k+q},
$$

l'hypothèse $\operatorname{tang}(\Lambda, \Gamma)=\operatorname{tang}\left(\Lambda^{\prime}, \Gamma\right)=D$, entraîne

$$
t=1-k+p=1-k+q
$$

d'où $p=q$. Alors

$$
\begin{aligned}
w^{\prime}-w & =\xi^{-q}\left(b^{\prime}(\xi, \eta)-b(\xi, \eta)\right) \frac{\partial}{\partial \eta} \\
& =\left[\xi^{-q}\left(b^{\prime}(\xi, \eta)-b(\xi, \eta)\right) \epsilon^{-1} \xi^{1-k^{\prime}}\right] \cdot v .
\end{aligned}
$$

Donc $f$ a, au plus, un pôle d'ordre $k^{\prime}-1+q$ sur $C$. Comme ce pôle est d'ordre $r k$ on en déduit

$$
r k \leqslant k^{\prime}-1+q=k+k^{\prime}+t-2 .
$$

d) Puisque $\operatorname{tang}\left(\Lambda_{\alpha}, \Gamma\right)=D$, on a que $\operatorname{Sing} \Lambda_{\alpha} \subset \operatorname{Supp}(D)$. Il suffit, donc, de prouver que si $Y$ est une composante irréductible de $\operatorname{Supp}(D)$, alors l'ensemble

$$
A_{Y}:=\left\{\alpha \in E(D): Y \subset \operatorname{Sing} \Lambda_{\alpha}\right\}
$$

est une sous-variété linéaire de $E(D)\left(\Lambda \notin A_{Y}\right)$.

Supposons $A_{Y}$ non-vide et soit $\alpha_{0} \in A_{Y}$. Alors $\alpha \in A_{Y}$ si et seulement si

$$
\left.\left((\lambda \circ \mu)_{*}(\alpha)-(\lambda \circ \mu)_{*}\left(\alpha_{0}\right)\right)\right|_{Y}=0 .
$$

C'est-à-dire $A_{Y}=\alpha_{0}+E_{Y}$ où

$$
E_{Y}:=\left\{\alpha \in E(D):\left.(\lambda \circ \mu)_{*}(\alpha)\right|_{Y}=0\right\},
$$

est un sous-espace vectoriel de $E(D)$.

Corollarre 5.5. - L'ensemble des feuilletages à singularités isolées $\Lambda^{\prime}$ de $X$ tels que $\Lambda^{\prime} \neq \Gamma$ et tang $\left(\Lambda^{\prime}, \Gamma\right)=D$ s'identifie naturellement $\grave{a}$ un sous-ensemble $\mathcal{F}_{\Lambda} \subset \mathbb{P}\left(\operatorname{Hom}\left(T, T_{X}\right)\right)$ dont l'adhérence $\overline{\mathcal{F}_{\Lambda}}$ est un sousespace linéaire de dimension égale à $\operatorname{dim}_{\mathbb{C}} E(D)$. En plus, les éléments de $\overline{\mathcal{F}_{\Lambda}}$ différents de $\Gamma$ qui n'appartient pas à $\mathcal{F}_{\Lambda}$ sont des feuilletages à singularités non-isolées et $\mathcal{F}_{\Lambda}$ est le complémentaire d'un nombre fini de sous-variétés linéaires dans $\frac{\mathcal{F}_{\Lambda}}{\text {. }}$. 
Preuve. - D'une part d'aprés le théorème 5.4 on sait que

$$
\overline{\mathcal{F}_{\Lambda}}=\mathbb{P}\left(\mathbb{C} \varphi+(\lambda \circ \mu)_{*}(E(D))\right) ;
$$

d'autre part, de l'injectivité de $(\lambda \circ \mu)_{*}$ suit

$$
\operatorname{dim}_{\mathbb{C}}\left(\mathbb{C} \varphi+(\lambda \circ \mu)_{*}(E(D))\right)=1+\operatorname{dim}_{\mathbb{C}} E(D) ;
$$

La première assertion du corollaire en résulte.

Si $\Lambda^{\prime} \in \overline{\mathcal{F}_{\Lambda}}$ alors, par ce qui précède, ou bien $\Lambda^{\prime}=\left(T, \varphi_{\alpha}\right)$ ou bien $\Lambda^{\prime}=\left(T,(\lambda \circ \mu)_{*}(\alpha)\right)$, pour un $\alpha \in E(D)$ convenable. Dans le premier cas, si $\Lambda^{\prime}$ est à singularités isolées, $\Lambda^{\prime} \in \mathcal{F}_{\Lambda}$ par le théorème 5.4a. Dans le deuxième cas, $\Lambda^{\prime}$ laisse invariantes les fibres de $h$. Alors si $\Lambda^{\prime} \neq \Gamma$, on a que $\Lambda^{\prime}$ est à singularités non-isolées. La dernière assertion découle de ceci et du théorème $5.4 \mathrm{~d}$.

Exemple 5.6. - Soit $E$ une courbe elliptique et soit $u \neq 0$ un champ de vecteurs holomorphe sur $E$. Considérons $X:=E \times E$ et dénotons $p_{1}, p_{2}$ : $X \rightarrow E$ les projections canoniques ; posons $B=E, h=p_{1}$. Finalement désignons par $\Lambda$ le feuilletage défini par $p_{2}$. Si on considère la famille des feuilletages de $X$ définis pas les champs de vecteurs $((1-t) u, t u)$ pour $0 \leqslant t \leqslant 1$, on constate que $\Gamma \in \overline{\mathcal{F}_{\Lambda}}$.

Exemple 5.7. - Considérons la même fibration de l'exemple 5.6. Soient $v_{1}=(u, 0), v_{2}=(0, u)$, des champs de vecteurs holomorphes sur $X$.

Fixons $q \in B$ et soit $F=h^{-1}(q)$. Soit $f \in \mathbb{C}(X), f \notin \mathbb{C}$, avec $\operatorname{div}_{\infty} f=$ $2 q$. Soit $\Lambda$ le feuilletage à singularités isolées défini par le champ méromorphe $v_{1}+f v_{2}(\operatorname{sur} X)$. Alors $\operatorname{tang}(\Lambda, \Gamma)=2 F$. On voit que tout $\Lambda^{\prime} \in \mathcal{F}_{\Lambda}$ est défini par un champ de la forme $v_{1}+g v_{2}, g \in \mathbb{C}(B) \backslash \mathbb{C}$, avec $\operatorname{div}_{\infty} g=2 q$. On en déduit que

$$
\operatorname{dim} \mathcal{F}_{\Lambda}=\operatorname{dim} \overline{\mathcal{F}_{\Lambda}}=2 .
$$

On vérifie que $\overline{\mathcal{F}_{\Lambda}}-\mathcal{F}_{\Lambda}$ est la réunion de deux droites dont l'une est formée par des feuilletages proportionnels à $\Gamma$ et l'autre est l'adhérence d'une famille de feuilletages transverses à $\Gamma$ en dehors de $F$, qui ont $F$ comme ensemble singulier.

\section{Calcul de $\operatorname{dim}_{\mathbb{C}} E(D)$}

On considère une fibration elliptique relativement minimale $h: X \rightarrow B$ de la surface analytique compacte et connexe $X$. On suppose que les fibres 
génériques de $h$ sont isomorphes, ce qui est bien le cas lorsqu'il existe sur $X$ un feuilletage turbulent de fibration adaptée $h$.

Comme au $\S 4$, pour tout $y \in B$ on note $X_{y}:=\sum_{j} k_{y j} C_{y j}$ la fibre de $y$, où les $C_{y j}$ sont les composantes irréductibles du support $h^{-1}(y)$ de $X_{y}$. De même $m_{y}$ est la multiplicité de $X_{y}$ et $k_{y j}^{\prime}:=k_{y j} / m_{y}$.

On se donne aussi un feuilletage turbulent $\Lambda$ de $X$ avec fibration adaptée $h$ et on note $D:=\operatorname{tang}(\Lambda, \Gamma)$ où $\Gamma$ est le feuilletage défini par $h$. Comme au $\S 5$ on écrit $D=\sum_{y, j} t_{y j} C_{y j}$ et on définit le diviseur effectif de $B$

$$
R:=\sum_{y \in B} r_{y} y, r_{y}=\inf _{j}\left[\frac{t_{y j}+k_{y j}+k_{y j}^{\prime}-2}{k_{y j}}\right],
$$

où le crochet indique la partie entière. Finalement on pose

$$
E(D)=H^{0}\left(B, \Omega_{B}(R) \otimes \mathcal{O}_{B} h_{*}\left(\mathcal{T}_{\Gamma}\right)\right)
$$

(voir définitions 5.1 et 5.2).

Le problème est de calculer $\operatorname{dim}_{\mathbb{C}} E(D)$.

Dans le résultat suivant on se sert de la classification de Kodaira des fibrations elliptiques relativement minimales (voir [1, chap. V, §7])

Lemme 6.1.- Les fibres singulières de $h$ ne sont jamais du type $I_{b}, I_{b}^{*}$ ou $m I_{b}$.

Preuve. - Il suit du fait que les fibres régulières de $h$ sont toutes isomorphes ([3, chap. $4, \S 3])$.

Corollaire 6.2. - Soit $\sigma$ le cardinal de l'ensemble $S$ des $y \in B$ tels que $h^{-1}(y)$ est une courbe singulière. Alors

$$
\chi(X) \leqslant \frac{5}{6} \sigma .
$$

Preuve. - Soit $e(X)$ la caractéristique d'Euler-Poincaré topologique de $X$. Puisque $K_{X} \cdot K_{X}=0$, d'après la formule de Kodaira ([1, chap. V, thm. (12.1)]), on a que $\chi(X)=e(X) / 12$ par la formule de Nöther ([1, chap. I, thm. (5.4)]). Si $h^{-1}(y)$ est une courbe non-singulière, alors $e\left(h^{-1}(y)\right)=0$ $([1$, chap. $\mathrm{V}, \S 7])$. On en déduit

$$
\begin{gathered}
e(X)=\sum_{y \in S} e\left(h^{-1}(y)\right) . \\
-407-
\end{gathered}
$$


Par ailleurs, si $y \in S$, de la classification de Kodaira suit que $e\left(h^{-1}(y)\right) \leqslant 10$ en tenant compte du lemme 6.1. Donc $e(X) \leqslant 10 \sigma$, ce qui complète la preuve.

LEMME 6.3. - Soit $y \in B$. Si $h^{-1}(y)$ est une courbe singulière, alors $r_{y}>0$.

Preuve. - Supposons d'abord $m_{y}>1$. On est dans le cas $m I_{1}$ de la classification de Kodaira. Comme $h^{-1}(y)$ est irréductible et contient un point singulier, elle est contenue dans le support de $D$. Donc $t_{y 1}>0$, d'où

$$
\frac{t_{y 1}+k_{y 1}+1-2}{k_{y 1}} \geqslant 1 \text {. }
$$

Supposons maintenant $m_{y}=1$. Si $k_{y j}>1$ on a

$$
\begin{aligned}
\frac{t_{y j}+k_{y j}+k_{y j}^{\prime}-2}{k_{y j}} & =\frac{t_{y j}+2 k_{y j}-2}{k_{y j}} \\
& \geqslant 2 \frac{k_{y j}-1}{k_{y j}} \\
& \geqslant 1
\end{aligned}
$$

Si $k_{y j}=1$, il résulte des considérations de [3, chap. 4, §3] que $C_{y j}$ est invariante par $\Lambda$. Donc $t_{y j} \geqslant 1$, d'où

$$
\frac{t_{y j}+k_{y j}+k_{y j}^{\prime}-2}{k_{y j}}=t_{y j} \geqslant 1 .
$$

LEMME 6.4. - On a $0 \leqslant \chi(X) \leqslant \operatorname{deg} R$ avec $\chi(X)=\operatorname{deg} R$ si et seulement si $\chi(X)=\operatorname{deg} R=0$.

Preuve. - En effet

$$
\operatorname{deg} R \geqslant \sigma \geqslant \frac{6}{5} \chi(X) .
$$

Les deux premières inégalités suivents du corollaire et du lemme ; la dernière résulte de la proposition (12.2) et remarque précédente de [1, chap. V] et de [1, chap. III, thm. (18.2)].

Notons $g$ le genre de $B$.

Proposition 6.5. - On a

$$
\operatorname{dim}_{\mathbb{C}} E(D)=\operatorname{deg} R+g-1-\chi(X),
$$

$\grave{a}$ moins que $\operatorname{deg} R=\chi(X)=0$. 
Preuve. - D'après le lemme 4.5 et le théorème de Riemann-Roch

$$
\begin{aligned}
\operatorname{dim}_{\mathbb{C}} E(D)= & \operatorname{dim}_{\mathbb{C}} H^{0}\left(B, \Omega_{B}(R) \otimes_{\mathcal{O}_{B}} h_{* 1}\left(\mathcal{O}_{X}\right)\right) \\
= & \operatorname{dim}_{\mathbb{C}} H^{0}\left(B, \Omega_{B} \otimes_{\mathcal{O}_{B}} \mathcal{O}_{B}(R) \otimes_{\mathcal{O}_{B}} h_{* 1}\left(\mathcal{O}_{X}\right)\right) \\
= & g-1+\operatorname{deg} R-\chi(X) \\
& \quad+\operatorname{dim}_{\mathbb{C}} H^{0}\left(B, \mathcal{O}_{B}(-R) \otimes_{\mathcal{O}_{B}} h_{* 1}\left(\mathcal{O}_{X}\right)^{\vee}\right),
\end{aligned}
$$

compte tenu du fait que deg $h_{* 1}\left(\mathcal{O}_{X}\right)^{\vee}=\chi(X)([1$, chap. V, Prop. (12.2)]).

Or, par le corollaire

$$
\operatorname{deg}\left(\mathcal{O}_{B}(-R) \otimes_{\mathcal{O}_{B}} h_{* 1}\left(\mathcal{O}_{X}\right)^{\vee}\right)=\chi(X)-\operatorname{deg} R<0,
$$

à moins que $\operatorname{deg} R=\chi(X)=0$, d'où l'assertion.

Proposition 6.6. - Supposons $\operatorname{deg} R=\chi(X)=0$. Alors $\operatorname{dim}_{\mathbb{C}} E(D)$ $=g$ ou $g-1$ selon que sur $X$ il existe ou pas de champ holomorphe de vecteurs tangents aux fibres de $h$ et jamais nul.

Preuve. - On observe que $R=0$ et on fait un calcul analogue à celui de la preuve de la proposition 6.5 ; on en déduit que $\operatorname{dim}_{\mathbb{C}} E(D)=g$ ou $g-1$ selon que $h_{* 1}\left(\mathcal{O}_{X}\right)$ soit ou ne soit pas isomorphe à $\mathcal{O}_{B}$. Mais $h_{* 1}\left(\mathcal{O}_{X}\right) \cong \mathcal{O}_{B}$ équivaut à l'existence d'un champ holomorphe de vecteurs sur $X$ tangent aux fibres de $h$ et jamais nul, d'après les lemmes 4.5 et 4.7 .

Remarque 6.7. - a) Rappelons que $\chi(X)=0$ implique que toutes les fibres de $h$ sont non-singulières (proposition (12.2) et remarque précédente de $[1$, chap. V] et $[1$, chap. III, Thm. (18.2)]).

b) $\chi(X)=0$ implique $\operatorname{deg} h_{* 1}\left(\mathcal{O}_{X}\right)=0$ ([1, chap. V, Prop. (12.2)]). Donc, si $g=0$ l'égalité $\chi(X)=0$ implique $h_{* 1}\left(\mathcal{O}_{X}\right) \cong \mathcal{O}_{B}$. C'est-à-dire, si $g=\chi(X)=0$ il existe un champ de vecteurs holomorphe sur $X$ jamais nul et tangent aux fibres de $h$.

Exemple 6.8.- Soit $E$ un tore de dimension 1. Fixons une involution sans points fixes $\imath$ de $E$. Considérons le quotient $X$ de $E \times E$ par l'opération de $\left(e_{1}, e_{2}\right) \mapsto\left(\imath\left(e_{1}\right),-e_{2}\right)$. La première projection $E \times E \rightarrow E$ passe au quotient et définit une fibration elliptique localement triviale $h: X \rightarrow B$. Le feuilletage de $E \times E$ défini par la deuxième projection $E \times E \rightarrow E$ passe au quotient et définit un feuilletage $\Lambda$ de $X$ transverse à $h$. Par la proposition 6.6

$$
\operatorname{dim}_{\mathbb{C}} E(D)=g-1=0 ;
$$

c'est-à-dire, $\Lambda$ est le seul feuilletage de $X$ transverse à $h$. 
Exemple 6.9. - (voir [3, chap. 4, §3]) Soit $E$ un tore de dimension 1. Notons $X$ une désingularisation minimale du quotient de $E \times E$ par l'involution $\left(e_{1}, e_{2}\right) \mapsto\left(-e_{1},-e_{2}\right)$. La première projection $E \times E \rightarrow E$ passe au quotient et définit une fibration elliptique $h: X \rightarrow B$, qui contient quatre fibres singulières $X_{y_{k}}$, pour $k=1,2,3,4$, toutes du type $I_{0}^{*}$ dans la classification de Kodaira, et dont la base est rationnelle. Il en résulte que $e(X)=24$ et $\chi(X)=2$. On note $\Lambda$ un feuilletage de $X$ avec fibration adaptée $h$.

Fixons un $y_{k}$ et écrivons

$$
X_{y_{k}}=2 C_{5}+\sum_{j=1}^{4} C_{j} ;
$$

posons $t_{j}:=t_{y_{k} j}$. Par construction chaque $C_{j}$ est invariante par $\Lambda$ ([3, chap. $4, \S 3])$. Donc (voir [3, chap. 2, Prop. 3])

$$
c_{1}\left(\mathcal{T}_{\Lambda}^{\vee}\right) \cdot C_{j}=-2+Z\left(\Lambda, C_{j}\right) \geqslant-1,1 \leqslant j \leqslant 4,
$$

car $Z\left(\Lambda, C_{j}\right) \geqslant 1$ par la formule de Camacho-Sad.

Si $C_{5}$ est invariante par $\Lambda$ on a de même

$$
c_{1}\left(\mathcal{T}_{\Lambda}^{\vee}\right) \cdot C_{5}=-2+Z\left(\Lambda, C_{5}\right) \geqslant 2,
$$

car chaque point d'intersection de $C_{5}$ avec l'une des $C_{j}$ est un point singulier de $\Lambda$.

D'autre part, si $C$ est une courbe irréductible contenue dans une fibre de $h$ on a

$$
\begin{aligned}
D \cdot C & =c_{1}\left(\mathcal{T}_{\Lambda}^{\vee}\right) \cdot C+c_{1}\left(\mathcal{N}_{\Gamma}\right) \cdot C \\
& =c_{1}\left(\mathcal{T}_{\Lambda}^{\vee}\right) \cdot C+c_{1}\left(\mathcal{T}_{\Gamma}^{\vee}\right) \cdot C-K_{X} \cdot C \\
& =c_{1}\left(\mathcal{T}_{\Lambda}^{\vee}\right) \cdot C+c_{1}\left(\mathcal{T}_{\Gamma}^{\vee}\right) \cdot C \\
& =c_{1}\left(\mathcal{T}_{\Lambda}^{\vee}\right) \cdot C-M \cdot C,
\end{aligned}
$$

d'après les lemmes $3.5 \mathrm{~b}$ et 3.3 , le lemme de Zariski ([1, chap. V, Thm. (12.1)]), le lemme 4.2 et la définition 4.1 .

On en déduit

$$
t_{5}-2 t_{j}=D \cdot C_{j} \geqslant-2,1 \leqslant j \leqslant 4
$$

et, lorsque $C_{5}$ est invariante,

$$
\sum_{j=1}^{4} t_{j}-2 t_{5}=D \cdot C_{5} \geqslant 4 .
$$


Alors, si $C_{5}$ n'est pas invariante $: t_{5}=0$ et $t_{j}=1,1 \leqslant j \leqslant 4$. Si, en revanche, $C_{5}$ est invariante, on obtient toute de suite que $t_{5}=2 t_{j}-2,1 \leqslant$ $j \leqslant 4$. Donc, en tous les cas

$$
t_{5}=2 t_{j}-2,1 \leqslant j \leqslant 4 .
$$

Finalement

$$
r_{y_{k}}=\frac{t_{5}+2}{2}
$$

Supposons $\Lambda$ transverse à toutes les fibres régulières de $h$. On obtient

$$
\operatorname{dim} \overline{\mathcal{F}_{\Lambda}}=\frac{a+2}{2}
$$

où on a posé $a:=\sum_{j=1}^{4} t_{y_{j} 5}$.

Observons que les $t_{y_{k}}(1 \leqslant j \leqslant 4)$ sont forcément pairs. Le cas où ceux-ci sont tous nuls résulte du feuilletage de $E \times E$ défini par la deuxième projection canonique.

\section{Existence de feuilletages turbulents}

Soit $h: X \rightarrow B$ une fibration elliptique relativement minimale de la surface analytique compacte et connexe $X$; comme avant $X_{y}$ désigne la fibre de $h$ au-dessus du point $y \in B$. On suposse donnés $y_{1}, \ldots, y_{n} \in B$ et, pour chaque $i=1, \ldots, n$, un voisinage ouvert $U_{i}$ de $y_{i}$ et un feuilletage à singularités isolées $\Lambda_{i}$ de $V_{i}:=h^{-1}\left(U_{i}\right)$, avec les conditions suivantes :

a) $U_{1} \cup \cdots \cup U_{n}=B$;

b) $X_{y}$ est régulière est transverse à $\Lambda_{i}$ pour tout $y \in U_{i}-\left\{y_{i}\right\}, 1 \leqslant i \leqslant n$;

c) si $X_{y_{i}}$ n'est pas régulière ou n'est pas transverse à $\Lambda_{i}$, alors $y_{i} \notin U_{k}$ pour $k \neq i$.

Posons

$$
X_{i}=X_{y_{\imath}}=\sum_{j} k_{i j} C_{i j},
$$

où les $C_{i j}$ sont les composantes irréductibles de $h^{-1}\left(y_{i}\right)$.

Soit $\Gamma$ le feuilletage associé à $h$ et soit $\Gamma_{i}=\Gamma \mid V_{i}(1 \leqslant i \leqslant n)$. On peut écrire alors

$$
\operatorname{tang}\left(\Lambda_{i}, \Gamma_{i}\right)=\sum_{\jmath} t_{i j} C_{i j} .
$$


Considérons le diviseur effectif de $X$

$$
D=\sum_{i, j} t_{i j} C_{i j}
$$

Soit $m_{i}$ la multiplicité de $X_{i}$ et posons $k_{i j}^{\prime}:=k_{i j} / m_{i}$. On définit le diviseur effectif $R:=\sum_{i=1}^{n} r_{i} y_{i}$ de $B$ avec

$$
r_{y}:=\inf _{j}\left[\frac{t_{y j}+k_{y j}+k_{y j}^{\prime}-2}{k_{y j}}\right],
$$

où le crochet indique la partie entière.

THÉORÈme 7.1. - Supposons que $\operatorname{deg} R>1+\chi(X)$. Alors, il existe un feuilletage turbulent $\Lambda$ de $X$, avec fibration adaptée $h$, tel que

$$
\operatorname{tang}(\Lambda, \Gamma)=D \text {. }
$$

Preuve. - Soit $y \in U_{i} \cap U_{k}$ avec $i \neq k$. Soit $u$ un champ de vecteurs holomorphe sur un voisinage $U \subset U_{i} \cap U_{k}$ de $y$. Alors $u$ se relève à des champs holomorphes $w_{i}, w_{k}$ sur $h^{-1}(U)$ tangents à $\Lambda_{i}, \Lambda_{k}$ respectivement. Donc, $w_{k}-w_{i}$ est tangent à $\Gamma$. On voit toute de suite que ceci définit un 1-cocycle $\left\{\xi_{i k}\right\}_{1 \leqslant i, k \leqslant n}$ du recouvrement $\left\{U_{i}\right\}_{1 \leqslant i \leqslant n}$ à valeurs dans $\Omega_{B} \otimes_{\mathcal{O}_{B}} h_{*}\left(\mathcal{T}_{\Gamma}\right)$. On peut aussi le supposer à valeurs dans $\mathcal{G}:=\Omega_{B}(R) \otimes_{\mathcal{O}_{B}} h_{*}\left(\mathcal{T}_{\Gamma}\right)$ par l'inclusion naturelle.

D'autre part, par hypothèse le degré du faisceau $\mathcal{G}$ est $>2 g-2$ ([1, chap. V, Pro. 12.2]); d'où $H^{1}(B, \mathcal{G})=0$, par la dualité de Serre.

On en déduit que, pour un choix convenable du recouvrement $\left\{U_{i}\right\}_{1 \leqslant i \leqslant n}$ il existe, pour chaque $i=1, \ldots, n$, une 1-forme méromorphe $\omega_{i}$ sur $U_{i}$, ayant, au plus, un pôle d'ordre $r_{i}$ en $y_{i}$, avec la propriété

$$
\left(\omega_{i} \otimes v_{i}-\omega_{k} \otimes v_{k}\right) \mid\left(U_{i} \cap U_{k}\right)=\xi_{i k},
$$

où $v_{i}$ est un champ holomorphe sur $V_{i}$ tangent à $\Gamma_{i}:=\Gamma \mid V_{i}$ et s'annulant sur $C_{i j}$ avec multiplicité $k_{i j}^{\prime}-1(1 \leqslant i \leqslant n)$; on a utilisé le lemme 4.7.

Maintenant nous allons définir un nouveau feuilletage $\Lambda_{i}^{\prime}$ de $V_{i}$ pour $1 \leqslant i \leqslant n$. Soit $w$ un champ de vecteurs holomorphe sur l'ouvert $V \subset V_{i}$ et tangent à $\Lambda_{i}$. Alors $\Lambda_{i}^{\prime} \mid V$ est défini par le champ de vecteurs

$$
w+h^{*}\left(\omega_{i}\right)(w) \cdot\left(v_{i} \mid V\right) .
$$


Un calcul direct montre que, d'après la définition de $r_{i}$, le champ de vecteurs $\omega_{i}(w) \cdot(v \mid V)$ est holomorphe. On en déduit que $\Lambda_{i}^{\prime}$ est bien défini et tel que

$$
\operatorname{tang}\left(\Lambda_{i}^{\prime}, \Gamma_{i}\right)=\operatorname{tang}\left(\Lambda_{i}, \Gamma_{i}\right),
$$

car $v_{i}$ est tangent à $\Gamma_{i}$.

Par ailleurs, soit $U \subset U_{i} \cap U_{k}(i \neq k)$ et soit $u$ un champ de vecteurs holomorphe sur $U$. Soient $w_{i}, w_{k}$ des relèvements de $u$ holomorphes sur $h^{-1}(U)$ et tangents à $\Lambda_{i}, \Lambda_{k}$ respectivement. Alors

$$
\begin{aligned}
w_{k}-w_{i} & =\omega_{i}(u) v_{i}-\omega_{k}(u) v_{k} \\
& =h^{*}\left(\omega_{i}\right)\left(w_{i}\right) v_{i}-h^{*}\left(\omega_{k}\right)\left(w_{k}\right) v_{k}
\end{aligned}
$$

sur $h^{-1}(U)$. Donc

$$
w_{i}+h^{*}\left(\omega_{i}\right)\left(w_{i}\right) v_{i}=w_{k}+h^{*}\left(\omega_{k}\right)\left(w_{k}\right) v_{k},
$$

sur $h^{-1}(U)$ d'où suit que $\Lambda_{i}^{\prime}$ et $\Lambda_{k}^{\prime}$ coïncident sur $U_{i} \cap U_{k}$. On en conclut que les $\Lambda_{i}^{\prime}$ se recollent et définissent un feuilletage $\Lambda$ de $X$ tel que

$$
\operatorname{tang}(\Lambda, \Gamma)=D \text {. }
$$

Si $\Lambda$ est à singularités isolées, on a fini. Sinon, observons que

$$
E(D)=\Omega_{B}(R) \otimes \mathcal{O}_{B} h_{*}\left(\mathcal{T}_{\Gamma}\right) \neq 0
$$

(proposition 6.5). Alors, comme dans le théorème 5.4, on peut associer à chaque $\alpha \in E(D)$ un feuilletage $\Lambda_{\alpha}$ de $X$ tel que tang $\left(\Lambda_{\alpha}, \Gamma\right)=\operatorname{tang}(\Lambda, \Gamma)$; le feuilletage $\Lambda_{\alpha}$ est à singularités isolées pour $\alpha$ convenable (théorème $5.4 \mathrm{~d}$ ).

\section{Bibliographie}

[1] Barth (W.), Peters (C.), VAN de Ven (A.). - Compact Complex Surfaces, Springer Verlag, (1984).

[2] Brunella (M.). - Feuilletages holomorphes sur les surfaces complexes compactes, Ann. Norm. Sup., $4^{a}$ sér., t. 30, p. 569-594 (1997).

[3] BRUnella (M.). - Birational Geometry of Surfaces, First Latin-Americam Congress, IMPA, (2000).

[4] Pan (I.), Sebastiani (M.). - Feuilletages tourbillonnés sur les fibrés principaux elliptiques, pré-publication.

[5] Gomez-Mont (X.). - Universal families of foliations by curves, Astérisque, 150-151, 109-129 (1987). 